\title{
Effect of Knotting on the Shape of Polymers
}

\author{
Eric J. Rawdon, ${ }^{\dagger}$ John C. Kern, ${ }^{\ddagger}$ Michael Piatek, ${ }^{\S}$ Patrick Plunkett, ${ }^{\prime \prime}$ Andrzej Stasiak,,$\perp$ \\ and Kenneth C. Millett"
}

\author{
Department of Mathematics, University of St. Thomas, St. Paul, Minnesota 55105; Department of \\ Mathematics and Computer Science, Duquesne University, Pittsburgh, Pennsylvania 15282; Department \\ of Computer Science and Engineering, University of Washington, Seattle, Washington 98195; \\ Department of Mathematics, University of California, Santa Barbara, Santa Barbara, California 93106; \\ Faculty of Biology and Medicine, Center for Integrative Genomics, University of Lausanne, Lausanne \\ CH 1015, Switzerland; and Department of Mathematics, University of California, Santa Barbara, \\ Santa Barbara, California 93106
}

Received June 20, 2008; Revised Manuscript Received September 4, 2008

\begin{abstract}
Momentary configurations of long polymers at thermal equilibrium usually deviate from spherical symmetry and can be better described, on average, by a prolate ellipsoid. The asphericity and nature of asphericity (or prolateness) that describe these momentary ellipsoidal shapes of a polymer are determined by specific expressions involving the three principal moments of inertia calculated for configurations of the polymer. Earlier theoretical studies and numerical simulations have established that as the length of the polymer increases, the average shape for the statistical ensemble of random configurations asymptotically approaches a characteristic universal shape that depends on the solvent quality. It has been established, however, that these universal shapes differ for linear, circular, and branched chains. We investigate here the effect of knotting on the shape of cyclic polymers modeled as random isosegmental polygons. We observe that random polygons forming different knot types reach asymptotic shapes that are distinct from the ensemble average shape. For the same chain length, more complex knots are, on average, more spherical than less complex knots.
\end{abstract}

\section{Introduction}

Ring polymer chains can be modeled as freely jointed random polygons. This simple representation of polymeric chains reflects their statistical properties under the so-called $\theta$-conditions, where independent segments of the polymer chains neither attract nor repel each other. ${ }^{1}$ Under $\theta$-conditions, linear polymers behave like ideal random walks and show scaling exponent $v=0.5$. If polymer chains are circular, the situation gets more complex as the scaling behavior depends on whether one studies all possible configurations or just those that represent a given topological type like unknotted circles. $^{2-11}$

It is an accepted convention in studies of shape and size of polymer chains to characterize actual configurations adopted by the polymers by calculating their inertial properties. Radius of gyration, i.e., the root-mean-square distance from the center of mass, is a standard measure of polymer size. In simulation studies, the mass of the polymer is assumed to be equally distributed among the vertex points of the simulated chains. Studies of overall polymer size reveal that the radius of gyration of circular polymers for a fixed knot type scales like that of self-avoiding walks ${ }^{2-4,12,13}$ with an estimated scaling exponent $v=0.5874 \pm 0.0002^{14}$ while phantom polymers behave like neutral ideal chains with the scaling exponent $v=0.5$.

Studies of shapes of polymer chains use the three principal moments of inertia calculated for given configuration of the chain to build an ellipsoid with the same ratio of its principal moments of inertia as those of the given polymer configuration. $\mathrm{Kuhn}^{15}$ was first to propose that the overall shape of random coils formed by polymer chains at thermodynamic equilibrium should, for entropic reasons, have the shape of a prolate ellipsoid. His proposal has been confirmed in numerical simula-

\footnotetext{
* Corresponding author. E-mail Andrzej.Stasiak@unil.ch.

University of St. Thomas.

$\div$ Duquesne University.

$\S$ University of Washington.

"University of California, Santa Barbara.

${ }^{\perp}$ University of Lausanne.
}

tion studies (see e.g. refs 9, 16, and 17) and also in experimental measurements. ${ }^{18,19}$ In the present study, we address how the shape and overall size of polymer chains are influenced by the presence of knots in these polymers.

The ellipsoid of inertia is defined using the moment of inertia tensor

$$
T_{i j}=\frac{1}{2 N^{2}} \sum_{n=1}^{N} \sum_{m=1}^{N}\left(X_{n}^{i}-X_{m}^{i}\right)\left(X_{n}^{j}-X_{m}^{j}\right) \quad(i=1,2,3 ; j=
$$

where $X_{n}^{i}$ denotes the $i$ th coordinate of the $n$th vertex and $N$ is the number of vertices in the polygon on which one has equally distributed the mass of the polymer. As $T_{i j}$ is a real symmetric tensor, it has three real eigenvalues $\lambda_{1}, \lambda_{2}$, and $\lambda_{3}$ giving the three principal moments of inertia and determining the corresponding eigenvectors providing the principal axes of inertia. The square roots of $\lambda_{1}, \lambda_{2}$, and $\lambda_{3}$ define the semiaxis lengths of the associated ellipsoid of inertia.

A critical question is how to best measure the spatial extent of this ellipsoid. To accomplish this objective, in 1986 Aronovitz and Nelson ${ }^{20}$ proposed a three-dimensional system, later modified by Cannon, Aronovitz, and Goldbart, ${ }^{21}$ which separates the size calculation (measured by the squared radius of gyration, see eq 4, which we will denote by $R$ ) from two shape descriptors: asphericity, $A$ (see eq 2), and nature of asphericity (see eq 3). In this definition, the asphericity and nature of asphericity are calculated using the three principal moments of inertia, i.e., the eigenvalues $\lambda_{1}, \lambda_{2}$, and $\lambda_{3}$ of $T_{i j}$. The asphericity was defined at the same time by Rudnick and Gaspari ${ }^{22}$ and has since become a principal target of study ${ }^{21,23-27}$ of those interested in assessing the spatial shape of polymers. For the sake of explicitness, we will refer to the nature of asphericity as the prolateness and denote it by $P$. Roughly speaking, the asphericity measures the degree to which the three axis lengths of the ellipsoid of inertia are equal. The prolateness indicates whether the largest or smallest axis length is "closer" to the 

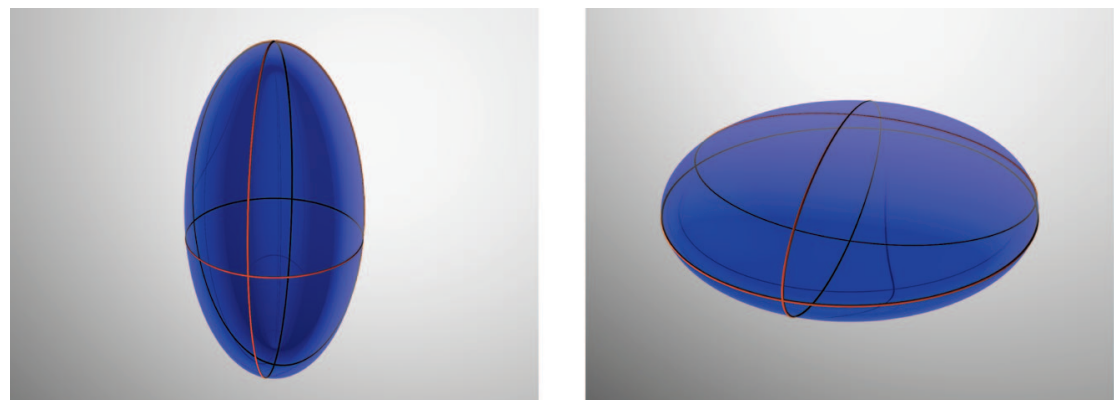

Figure 1. Examples of prolate (left) and oblate (right) ellipsoids. In a prolate ellipsoid, the most round equatorial ellipse is perpendicular to the longest axis, e.g., a rugby ball. In an oblate ellipsoid, the shortest axis is perpendicular to the most round equatorial ellipse, e.g., M\&M candy. The semiaxis lengths for the ellipsoids shown are $(1,0.5,0.5)$ and $(1,1,0.4)$, respectively. The asphericity of both ellipsoids above is 0.0625 , and the prolateness values are 1 and -1 , respectively.

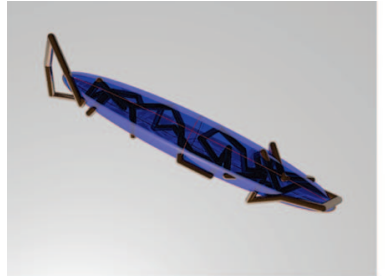

(a) $R \approx 7.08$

$A \approx 0.35$

$P \approx 0.99$

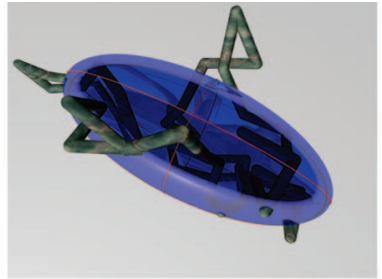

(b) $R \approx 4.03$

$A \approx 0.068$

$P \approx 0.67$

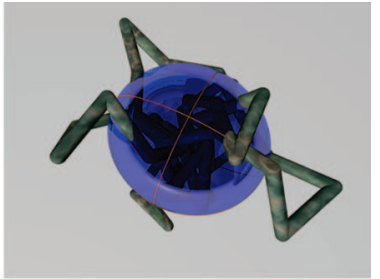

(c) $R \approx 1.73$

$A \approx 0.0012$

$P \approx-0.52$

Figure 2. Examples of 50 edge polygonal trefoil knots with high, medium, and low asphericity shown with their characteristic inertial ellipsoids.

middle axis length and takes values between -1 and 1 , thereby quantifying the transition from oblate to prolate shapes.

Together, the squared radius of gyration, asphericity, and prolateness form an independent set of parameters describing an ellipsoid. In section 2, we discuss relationships between $R$, $A$, and $P$ and explain why we have chosen to replace the eigenvalues employed in their definition by the square root of 3 times the eigenvalues, i.e., the semiaxis lengths of the characteristic inertial ellipsoid, in our study of the shape of polymers. We report these new measures of asphericity and prolateness as well as those used previously.

Several studies have addressed the question of how the asymptotic value for the asphericity depends on the solvent quality. In contrast to linear chains that show large differences in asphericity depending on whether the chains are self-avoiding (good solvent) or not ( $\theta$-solvent), the circular chains show quite similar asymptotic values of asphericity under these two different conditions. ${ }^{25}$ Diehl and Eisenriegler ${ }^{26}$ determined theoretically (using the eigenvalues or, equivalently, squared axis lengths) that the asymptotic value of asphericity for non-self-avoiding random polygons is 0.2464 . Simulation studies by Bishop and Saltiel indicated that for self-avoiding polygons the asphericity reaches an asymptotic value of $0.255 \pm 0.010 .{ }^{25}$ More recently, Zifferer and Preusser have simulated self-avoiding ring polymers with up to 8192 segments and, upon extrapolation to infinite chain length, concluded that the asymptotic value of asphericity for this system is $0.2551 \pm 0.0005 .{ }^{16}$ One of the goals of this paper is to estimate numerically this asymptotic value for polygons with a fixed knot type.

When a polygon has between three and five vertices, the only knot possible is the unknot, i.e., a polygon that is topologically equivalent to a circle. At six edges, the first nontrivial knot appears, the trefoil knot, known as 3 . For increasing numbers of edges, more and more different (and more complicated) types of knots become possible (see e.g. refs 28-30 for a discussion of the minimal number of edges in an equilateral polygon required to realize each knot type and the growth of the number of knot types possible as a function of the number of edges). In addition, the probability of obtaining a knotted polymer tends to one as the length goes to infinity. ${ }^{31-34}$

Here we investigate the shape of circular polymers with fixed knot type, as measured by their asphericity and prolateness, and determine the dependence of shape on the length and knotting of the polymer. We find that for "small" numbers of edges polymers of a fixed nontrivial knot type tend to be more spherical (lower asphericity) than phantom polygons with the same number of edges. However, the opposite is true for very long knotted chains that, at least in case of the knot types we analyzed, become less spherical than phantom polygons (see Figures 6 and 8). Prolateness shows a more complex behavior: short knotted chains of a given nontrivial knot type are initially less prolate than phantom polygons of the same size. Then, in the intermediate size range, the knotted chains become more prolate than the corresponding phantom chains. Finally, in the long chain regime, we again observe that knotted chains become less prolate than phantom chains (see Figures 7 and 8).

\section{Exploration of Shape Measures}

As discussed in the previous section, we use a slight variation of asphericity and prolateness to measure the shape of individual ellipsoids describing circular polymers as we replace the principal moments of inertia, i.e., eigenvalues of the moment of inertia tensor, by the square root of 3 times the eigenvalues. To explain why this is helpful, we first review the principal concepts: the asphericity is a number between 0 (implying a spherical shape when $a=b=c$ ) and 1 (implying a rodlike shape when $b=c=0$ ) and is defined by

$$
A(a, b, c)=\frac{(a-b)^{2}+(a-c)^{2}+(b-c)^{2}}{2(a+b+c)^{2}}
$$

where $a, b$, and $c$ are measurements of the size of the ellipsoid of inertia. The prolateness has values between -1 (perfectly oblate, e.g. when $a=b>c$ ) and 1 (perfectly prolate, e.g. when $a>b=c$ ) and is defined by 


$$
P(a, b, c)=\frac{(2 a-b-c)(2 b-a-c)(2 c-a-b)}{2\left(a^{2}+b^{2}+c^{2}-a b-a c-b c\right)^{3 / 2}}
$$

To get a sense for the values of prolateness, assume that $a \geq b$ $\geq c \geq 0$. Then $P(a, b, c)=0$ when $b=(a+c) / 2$. When $b<$ $(a+c) / 2$, the prolateness is positive (i.e., the ellipsoid is prolate), and when $b>(a+c) / 2$, the prolateness is negative (i.e., the ellipsoid is oblate). Note that both $A$ and $P$ are invariant of scale and symmetric in $a, b$, and $c$.

As discussed earlier, traditionally the principal moments of inertia $\lambda_{1}, \lambda_{2}$, and $\lambda_{3}$ have been used as the arguments $a, b$, and $c^{20-27}$ The scaling arguments used to predict asymptotic values for random walks, random polygons, star shapes, etc., use these eigenvalues as well. Cannon et al. ${ }^{21}$ observed that the asphericity is "biased towards larger configurations". However, with additional care in the definitions, one can eliminate the bias of $R$ in $A$ while preserving the unbiased nature of $P$.

One of the goals in defining the asphericity and, subsequently, the nature of asphericity or prolateness is to have true measures of shape that are unbiased by the size of the polygon. A standard measure of the size of a polymer, the squared radius of gyration, is determined from its moment of inertia tensor as the sum of its three eigenvalues:

$$
R=\lambda_{1}+\lambda_{2}+\lambda_{3}
$$

With the objective of eliminating the scale bias, we first define an ellipsoid whose principal moments of inertia and principal axes coincide with those of the polygon. This ellipsoid has semiaxis lengths of $\alpha_{i}=\left(3 \lambda_{i}\right)^{1 / 2}, i=1,2,3$. We refer to this ellipsoid as the characteristic inertial ellipsoid (see Figure 2 where one can observe the relationship between polygons and their associated characteristic inertial ellipsoids).

The characteristic inertial ellipsoid has the attractive property that the characteristic inertial ellipsoid of an ellipsoid is itself. This is consistent with the definition of the radius of gyration, for example, where a sphere of radius $r$ has radius of gyration equal to $r$. In contrast, the ellipsoid of inertia of an ellipsoid has the same principal axes of inertia, but the semiaxis lengths are scaled by the factor $1 / \sqrt{ } 3$; i.e., the ellipsoid of inertia is a shrunken version of the original.

For the characteristic inertial ellipsoid, the squared radius of gyration is

$$
R(a, b, c)=\frac{a^{2}+b^{2}+c^{2}}{3}
$$

where we use the arguments $\alpha_{1}, \alpha_{2}$, and $\alpha_{3}$, i.e., the semiaxis lengths of the characteristic inertial ellipsoid.

While this might appear to be a more complex way to compute $R$, it is really $A$ and $P$ that have been changed to ensure that the triple, $R, A$, and $P$, are independent and unbiased as demonstrated by their gradients which are now orthogonal:

$$
\nabla R \cdot \nabla A=\nabla R \cdot \nabla P=\nabla A \cdot \nabla P=0
$$

where $\cdot$ is the standard dot product in $\mathbb{R}^{3}$. Equivalently, one observes that their contour surfaces now provide an orthogonal system (see Figure 3). One way of viewing this distinction is via an analogy with bases for vector spaces and the special properties enjoyed by orthogonal bases or sets of functions such as Legendre polynomials, Bessel functions of the first kind, or the family $\{\sin (n x), \cos (n x)\}$ leading to Fourier series. The latter are not only bases but provide orthogonal independent measures expressing the structure of the vector space.

In constrast, if one were to use the eigenvalues instead of the axis lengths, this results in redefining $R$ to be $R(x, y, z)=x$ $+y+z$ where the arguments are now $\lambda_{1}, \lambda_{2}$, and $\lambda_{3}$. The

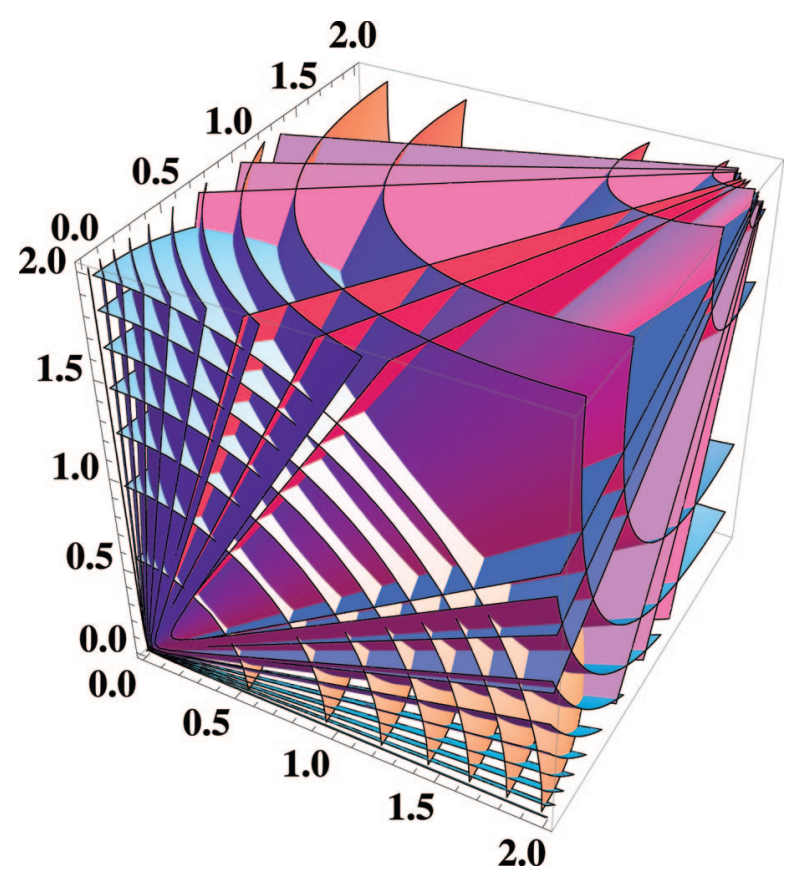

Figure 3. When using the semiaxis lengths for the parameters of $R, A$, and $P$, a Mathematica ${ }^{35}$ plot of the contour surfaces shows that they intersect perpendicularly (due to the fact that $\nabla R \cdot \nabla A=\nabla R \cdot \nabla P=$ $\nabla A \cdot \nabla P=0$ ). The $x, y$, and $z$ axes shown are the semiaxis lengths of ellipsoids and, for the sake of this figure, range from 0 to 2 . The contour surfaces are the semiaxis lengths corresponding to some fixed $R, A$, or $P$ values.

Table 1. Estimates of the Asymptotic Values for Asphericity

\begin{tabular}{lcc}
\hline knot & axes & eigenvalues \\
\hline phantom & $0.074360 \pm 0.00042$ & $0.2461 \pm 0.0013$ \\
$0_{1}$ & $0.07875 \pm 0.00074$ & $0.2550 \pm 0.0023$ \\
$3_{1}$ & $0.07931 \pm 0.0010$ & $0.2561 \pm 0.0030$ \\
$4_{1}$ & $0.0797 \pm 0.0021$ & $0.2569 \pm 0.0066$ \\
$5_{1}$ & $0.0814 \pm 0.0037$ & $0.263 \pm 0.011$ \\
$5_{2}$ & $0.0819 \pm 0.0029$ & $0.2643 \pm 0.0085$ \\
$6_{1}$ & $0.0853 \pm 0.0055$ & $0.275 \pm 0.016$ \\
$6_{2}$ & $0.0807 \pm 0.0051$ & $0.259 \pm 0.016$ \\
$6_{3}$ & $0.0782 \pm 0.0063$ & $0.254 \pm 0.020$
\end{tabular}

definitions of $A$ and $P$ remain the same. In such a case, we still obtain $\nabla R \cdot \nabla P=\nabla A \cdot \nabla P=0$. However, $\nabla R \cdot \nabla A=[6(x y+$ $\left.\left.x z+y z-x^{2}-y^{2}-z^{2}\right)\right] /(x+y+z)^{3}$, thereby showing the inherent bias in the system.

In order to employ unbiased measures of shape, we will use $R$, $A$, and $P$ with the semiaxis lengths of the characteristic inertial ellipsoid $\alpha_{1}, \alpha_{2}$, and $\alpha_{3}$ as arguments. As a consequence, the specific numerical results differ from previous theoretical and numerical studies. However, we present both values in Table 1 and observe that our data provide estimates using the eigenvalues which are consistent with those found in earlier studies. Note that due to the scale invariance of $A$ and $P$ (using either the eigenvalues or the semiaxis lengths) and the fact that the semiaxis lengths of ellipsoid of inertia and characteristic inertial ellipsoid differ by a common factor of $1 / \sqrt{ } 3$, the ellipsoid of inertia and characteristic inertial ellipsoid share the same $A$ and $P$ values.

The asphericity and prolateness, together, give a quantification of the shape of the polymer (see Figures 6-8). The dominant factor is the asphericity which measures the degree to which the three eigenvalues of the inertial tensor are equal. For example, an ellipsoid with semiaxis lengths 1,1 , and 0.4 has asphericity equal to 0.0625 and prolateness equal to -1 . In contrast, with the same asphericity of 0.0625 , one has the other extreme, a prolate ellipsoid with one axis of length 1 and two axes of length $1 / 2$, giving a prolateness equal to +1 (see Figure 
1). When the asphericity is very close to zero, i.e., the semiaxis lengths are almost equal, the variation between the most and least prolate shapes is extremely small as the shape is constrained by the asphericity. For example, an ellipsoid with semiaxis lengths 1,1 , and 0.99 has $A \approx 1.13 \times 10^{-5}$ and $P=$ -1 while an ellipsoid with semiaxis lengths $1,0.99$, and 0.99 has $A \approx 1.12 \times 10^{-5}$ and $P=1$.

Therefore, the asphericity provides a first-order measurement of the shape of the polymer, and prolateness is a second-order descriptor of how the asphericity value is attained. Note that for a given asphericity value not all prolateness values are possible. For example, a rodlike shape has asphericity close to 1 , thereby forcing the prolateness to take values close to 1 as well. In fact, a prolateness of -1 is possible only with shapes where $A \leq 1 / 4$.

\section{Computations}

We have analyzed equilateral random polygons from 6 to 48 edges with a step size of 2 and from 50 edges to 500 edges by a step size of 10 edges. For each number of edges, we generated 400000 random knots using the hedgehog method. ${ }^{36}$ To identify the knot type of each of the polygons, we calculated the HOMFLY polynomial ${ }^{37}$ using the program of Ewing and Millett. $^{38}$ As the HOMFLY polynomial is not faithful to the knot type (i.e., there exist knots which are distinct but which have the same HOMFLY polynomial), we actually determine the distribution of HOMFLY polynomials of the random polygons and employ this as a surrogate for the knot type. However, the probability of finding other knots with the same HOMFLY polynomials as the simple knot types analyzed here is orders of magnitude lower than the probability of these simple knot types. Therefore, Figure 5 gives a faithful presentation of the probabilities of knots studied here. This set of random knots was also used in refs 10 and 11, and a more detailed description of the generation method can be found there.

We calculate the asphericity and prolateness for each of the random knots and keep a running list of the asphericity and prolateness values for the given HOMFLY polynomial with the given number of edges. Average asphericity and prolateness values are then computed for the knots $0_{1}, 3_{1}, 4_{1}, 5_{1}, 5_{2}, 6_{1}, 6_{2}$, and $6_{3}$ and for the entire knot population (i.e., phantom polygons) at each number of edges. Because the average asphericity and prolateness for the two versions of chiral knots will be the same, we combine those data sets. For example, $+3_{1}$ and $-3_{1}$, the right- and left-handed versions of the trefoil knot, are combined into a common $3_{1}$ file to provide more robust data. The other chiral knots in this study are $5_{1}, 5_{2}, 6_{1}$, and $6_{2}$.

One of the goals of this research is to describe the asymptotic shape of knotted and phantom polygons. To this end, we have estimated the asymptotic values of the asphericity, for both the axis length and the eigenvalue definitions, for the different classes of polygons. We used a Monte Carlo Markov chain analysis, described in more detail in ref 11 and its Supporting Information, to compute $95 \%$ confidence intervals for the asymptotic value. The fitting function ${ }^{27} A+B / \sqrt{ } x+C / x$ is used and applied to the data for 100 edges and larger, wishing to minimize small edge effects. For a fixed number of edges, the asphericity values are not normally distributed for a given knot type nor for the phantom polygons, so the grouping procedure described in ref 11 was used. Gibbs sampling ${ }^{39}$ was utilized to minimize computation time. In the end, we computed 3500 likely fitting functions for the phantom polygons and each of the knot types. The values shown in Table 1 correspond to the mean and $95 \%$ confidence ranges for the value of $A$ in the likely fitting functions.

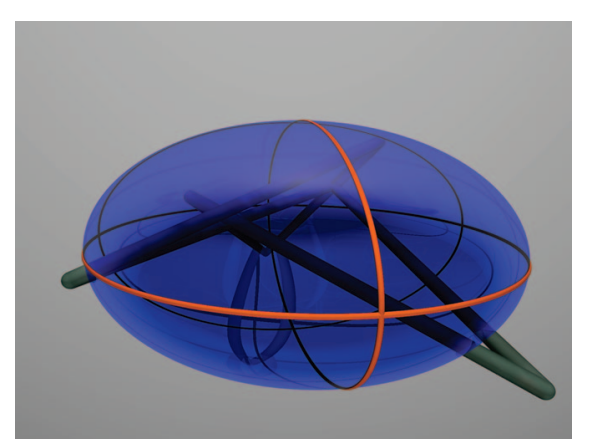

Figure 4. A hexagonal equilateral $3_{1}$ is shown with $A \approx 0.05$ and $P \approx$ -0.3 , the mean values for 6 -edge trefoils. Because the minimum number of edges required to construct a trefoil is 6 , the configurations tend to be nearly planar, forcing the characteristic inertial ellipsoid to be oblate. The thickened polygon and ellipsoid are shown from a position slightly off the longest principal axis.

\section{Asphericity and Prolateness of Knotted Polymers}

The random polygons were divided into the individual knot types, and their shapes were analyzed in terms of asphericity and prolateness. Figures $6-8$ show how the average asphericity and prolateness depend on the knot type and the size of the polygon. It is interesting to analyze some of these profiles in order to understand better their meaning. The asphericity profile for unknots (see Figure 6) shows that for small number of segments (say 6 and 8 segments) the unknotted polygons deviate strongly from spherical symmetry. However, the asphericity values in this range do not tell us whether the polygons are aspherical due to adopting discoidal planar configurations or due to forming very elongated shapes. Inspection of Figure 7 reveals, however, that unknotted polygons with 6 or 8 segments have on average positive prolateness. Therefore, we can conclude that the dominant deviation from spherical symmetry for unknotted polygons with small number of segments is toward forming elongated configurations. This contrasts with the negative prolateness of polygons with 6 segments that form trefoil knots (see Figure 7) and have on average an oblate shape (negative prolateness). In fact, isosegmental hexagons forming trefoil knots are quite restricted in their freedom to change shapes and adopt rather regular planar configurations. Figure 4 shows a hexagonal trefoil with a typical shape $(A \approx 0.05, P \approx$ $-0.3)$ together with its characteristic inertial ellipsoid. However, polygons forming trefoil knots with increasing number of segments quickly become prolate on average (see Figure 7), and their asphericity increases (see Figure 6).

A more general comparison of the asphericity of polygons forming different knot types reveals that for a given number of segments the polygons forming more complex knots are on average more spherical, i.e., have lower asphericity, than polygons forming less complex knots (see Figure 6). We expect however that for very long polymers the asphericity values of various simple knots will approach the same universal value. A general comparison of prolateness of polygons forming various knot types (see Figure 7) reveals that, for a given number of segments, the prolateness of less complex knots is lower than that of more complex knots. It is interesting to note that for the individual knot types analyzed here the prolateness reaches its maximal value for relatively short polygons $(n<70)$ and then shows a decrease. It may seem contradictory that the decrease in prolateness with the increasing chain length is associated with increasing asphericity (compare Figures 6-8). However, there is no real contradiction as the flattening of a rugby ball shape from its sides decreases its prolateness and increases its asphericity.

After exploring the asphericity and prolateness profiles for polygons forming individual knot types, let us analyze the 


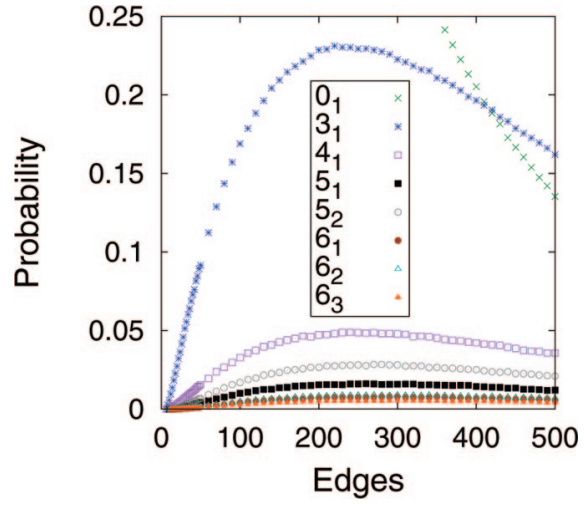

Figure 5. Relative probabilities of obtaining the knot types through six crossings.

corresponding profiles for the ensemble average of all polygons grouped together. Such a statistical set represents phantom polygons that can freely undergo intersegmental passages such as those exemplified by circular DNA molecules in the presence of type II DNA topoisomerase. Of course, the profile of all polygons is the weighted average of profiles for individual knot types where the relative probability of a given knot type is taken into account. Therefore, for very small number of segments, where unknots dominate, the profile for phantom polygons closely follows that of the unknots. As polygon sizes increase and nontrivial knots become frequent, the asphericity and prolateness of phantom polygons rapidly approach their respective characteristic constant values.

As discussed earlier, our asphericity definition is different from the one used in a number of previous studies of polymer shapes. ${ }^{16,25,26}$ However, for the purpose of comparison, we have also used the traditional definition. Since the number of tested configurations was highest for phantom polygons, that statistical sample provides us with the highest accuracy in the asphericity asymptote estimation. Using that sample, we have obtained the value of $0.2461 \pm 0.0013$ (see Table 1) as the asymptotic value of asphericity for non-self-avoiding random polygons. Our numerical estimation shows a perfect agreement with the previously reported theoretically predicted value of 0.2464 for this type of polygons ${ }^{26}$ and outperforms in this respect some earlier numerical studies. ${ }^{24}$

Our second best statistical sample consists of unknotted polygons. For the asymptotic value of the asphericity for polygons forming unknotted rings, we have obtained the value of $0.2550 \pm 0.0023$ (see Table 1). This value is essentially the same as those obtained in earlier simulation studies investigating the asphericity of polygons with excluded volume $(0.255 \pm$ $0.010^{25}$ and $\left.0.2551 \pm 0.0005\right) .{ }^{16} \mathrm{It}$ is important to stress here that in our study the modeled polygons had their effective diameter set to zero. However, if one investigates how various statistical properties depend on the polygon size while maintaining the same knot type, this is equivalent to introducing topological excluded volume. ${ }^{3}$ The topological excluded volume has been shown to behave like the standard excluded volume when analyzing the scaling of overall dimensions of polygons. ${ }^{2,5,7}$ The results presented here indicate that both the standard and topological excluded volumes affect universal shape descriptors, e.g., the asphericity, in the same way. Our statistical samples for individual knot types decrease with the complexity of the knot type (see Figure 5), and this increases the error in estimating the asymptotic value of the asphericity for modeled polymers forming a given knot type (see Table 1). However, our data are consistent with the hypothesis that all individual knot types reach the same asymptotic value, a value that is characteristic of self-avoiding polygons, although the speed with which the asymptotic value is reached decreases with the complexity of the knot (see Figure 6). This hypothesis also is consistent with observations that, on average, as the polymer length increases, the knotted portion of the chain gets smaller in comparison to the overall length of the chain, and therefore, the large unknotted portion of the polymer dominates the average configuration. ${ }^{40-42}$ There is numerical evidence that knotting is, on average, weakly local. ${ }^{43}$ Thus, the influence of simple knots, such as a single trefoil, on the average shape is quite small asymptotically. From another perspective, although it has been shown that global knotting is also present in large polymers, ${ }^{44}$ it appears that this presence is quite rare and does not contribute substantially to the average measures for a given knot type or for that of the phantom polygons.

\section{Size and Shape of Knotted Polymers}

We have concentrated on scale independent measures of overall shape adopted by modeled polymers like asphericity and prolateness. However, size also matters, and to completely describe inertia preserving ellipsoids that characterize the shapes of knotted polymers with a given length, one needs to consider the absolute sizes of these ellipsoids, where the natural size measure is the statistical segment length. Figure 9 presents the characteristic inertial ellipsoids for the average shapes of the knots $0_{1}, 3_{1}$, and $4_{1}$ and also of phantom chains formed by 500 edge polygons. This form of presentation (nested ellipsoids) allows visual comparison of average shapes of polygons with different topology. We can see that the ellipsoid characterizing unknots forms the external shell and therefore is bigger than ellipsoids characterizing nontrivial knots. As the knots get more complicated, the ellipsoids representing them become smaller. However, they maintain very similar aspect ratios, and it is hardly visible that $4_{1}$ knots are on average more spherical than unknots (see Figure 6). The most internal shell in Figure 9 represents phantom polygons as these have the smallest overall dimensions from this set of knots. However, more complex knots, e.g., the $10_{165}$ knot, would be smaller than phantom knots for polygons with 500 edges.

The situation presented in Figure 9 illustrates the particular case of 500 edge polygons. What would be the corresponding image for very long chains? We conjecture that for such a situation the nested ellipsoids would be very closely spaced, like onion skins. The external skin would be still that of the unknot, and the sequential skins would be ordered according to the complexity of the knot $3_{1}, 4_{1}$, five crossing knots, six crossing knots, etc. Toward the center of the onion, one would have extremely complex knots, while the skin representing the average size of phantom knots would be placed between the external skin representing unknots and the internal skins representing most complex knots possible for this size of the polygon. We also conjecture that the external skins (i.e., ellipsoids) representing simple prime knots would all be asymptotically close to the aspect ratio attained by the ellipsoid representing unknots, while very complex knots would be more spherical. At this point, we are uncertain whether the order of the skins for all knots will be the same for all chain sizes, i.e., whether there could be an example of two knot types where one would have its overall dimensions smaller than the other at 500 segments, for example, but not at 1000 segments. However, it is probably safe to conjecture that the order of skins (ellipsoids) representing knots belonging to the same family of knots (like simple torus knots $3_{1}, 5_{1}, 7_{1}$, etc.) will always follow the order of the minimal crossing number, provided that the number of segments in the polygon is significantly bigger than the minimal number of segments needed to form most complex knots under consideration. 

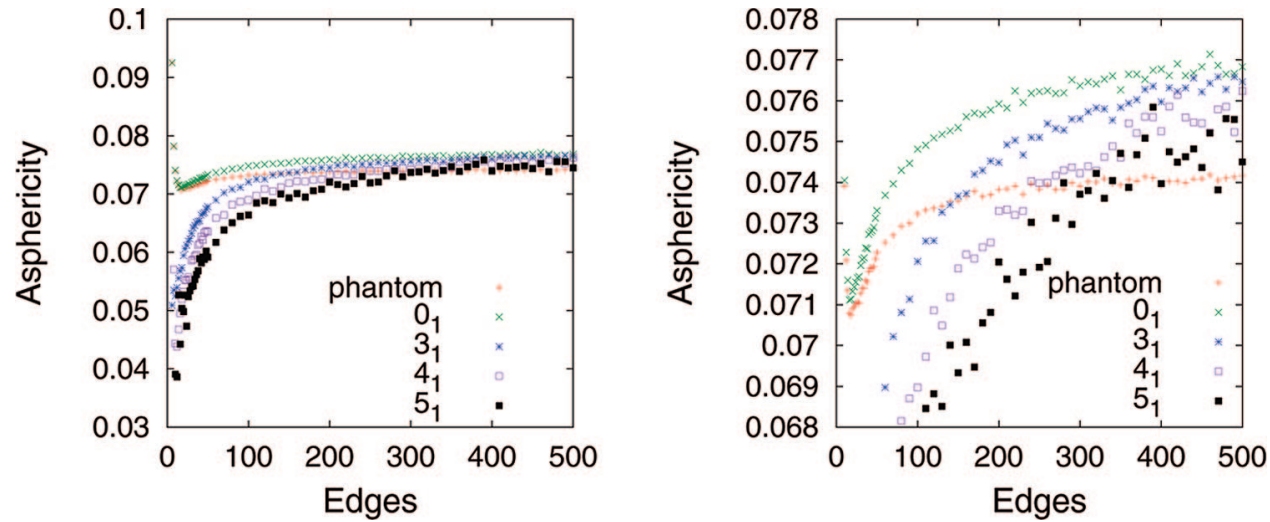

Figure 6. Scaling profiles for the average asphericity of knotted polygons and phantom polygons. In the right panel, we restrict the vertical dimension to show more clearly the differences between individual knot types.
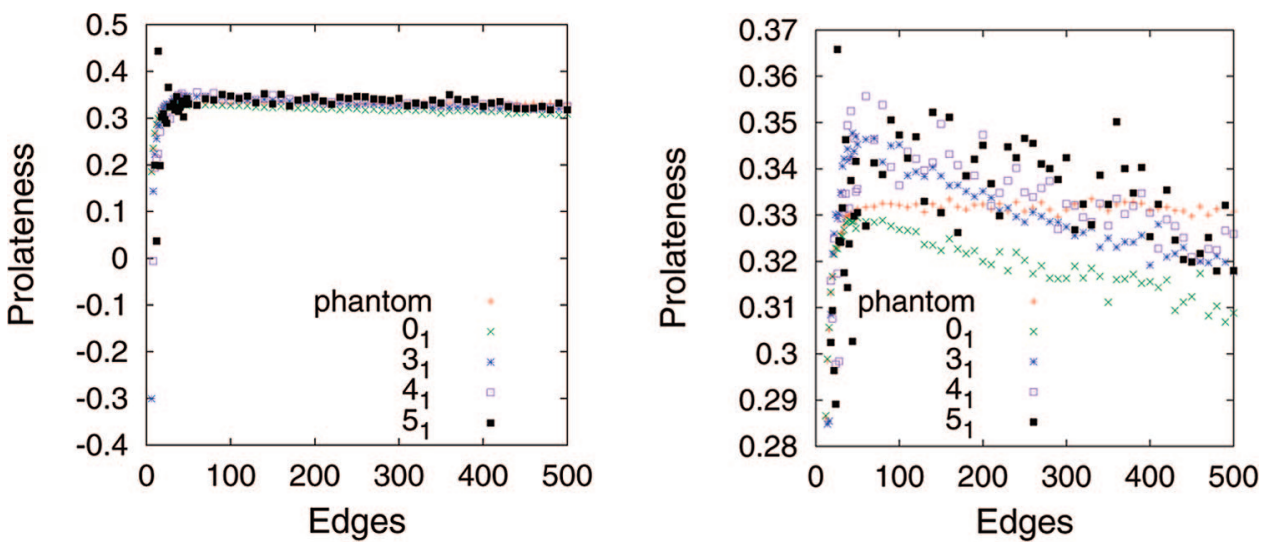

Figure 7. Scaling profiles for the average prolateness of knotted polygons and phantom polygons. In the right panel, we restrict the vertical dimension to show more clearly the differences between individual knot types.
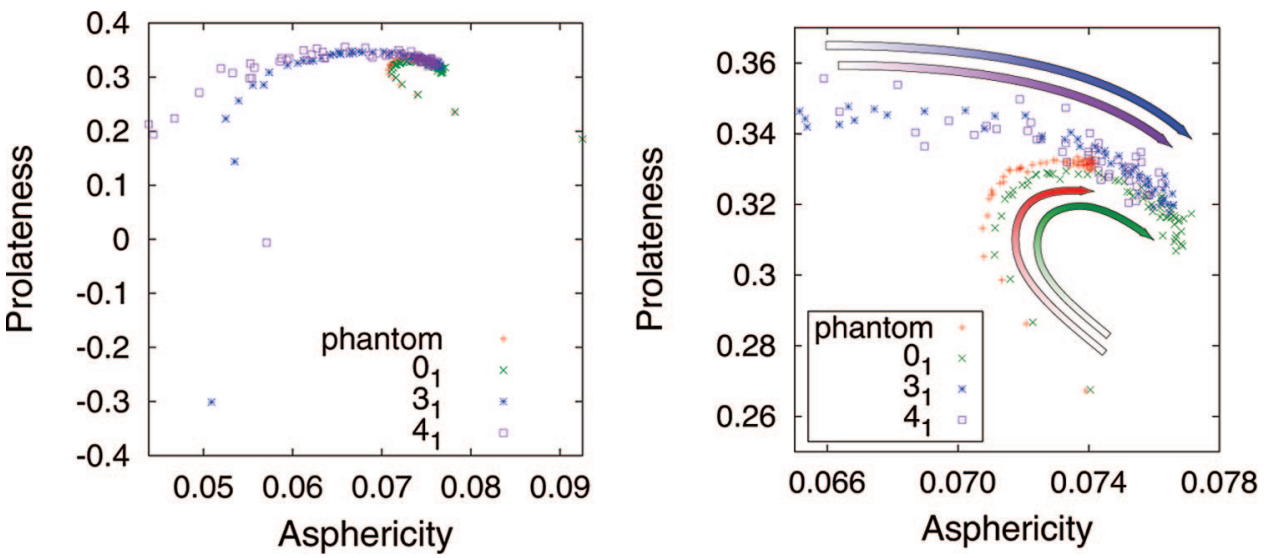

Figure 8. Average asphericity and prolateness for phantom polygons, $0_{1}, 3_{1}$, and $4_{1}$ knotted polymers with increasing length. The arrows show the direction of increasing numbers of edges. In the right panel, we restrict the vertical dimension to show more clearly the differences between individual knot types.
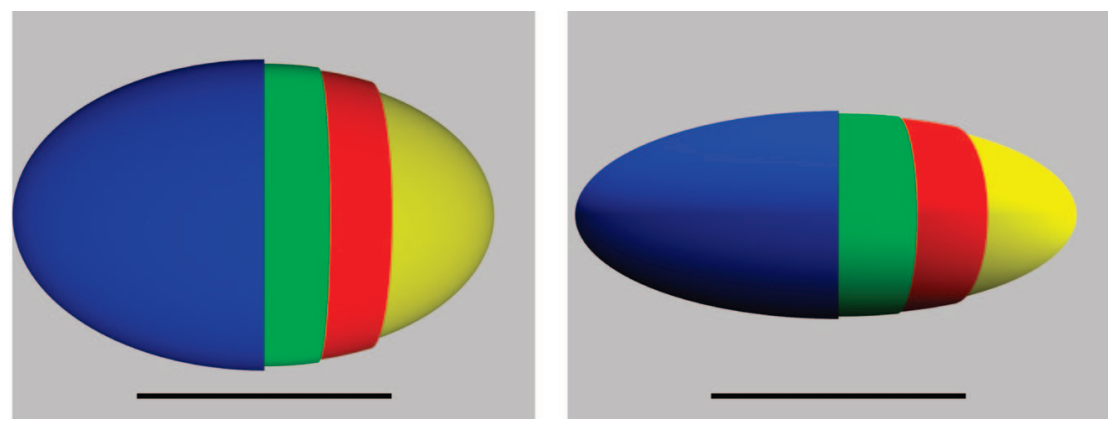

Figure 9. Average ellipsoids for 500 edge $0_{1}$ (in blue), $3_{1}$ (in green), $4_{1}$ (in red), and phantom polygons (in yellow) as seen along the two shortest axes of inertia. The black bar below the ellipsoids represents the size of 10 statistical segments. 


\section{Conclusions}

The notion that the overall shape of randomly fluctuating polymeric molecules can be approximated by prolate ellipsoids rather than by spheres was published in 1934 by Kuhn. ${ }^{15}$ Over the years theoretical and numerical studies have established that as the chain size tends to infinity, the asphericities of ellipsoids describing the inertial properties of modeled polymers asymptotically approach characteristic constant values. $9,16,17,20-22,24-26$ These values are known to be different for linear and circular chains and are, in addition, influenced by the solvent quality. Here we have provided unbiased measures of inertial shape and established that the topology of the chains also affects their overall shape. We have shown that for a fixed chain size the modeled polymer molecules forming less complex knots are, on average, more spherical than configurations of more complex knotted chains. Furthermore, for each knot type, there is a chain length starting with which polygons representing this knot type will be on average less spherical than the average shape of phantom polygons for every number of segments beyond this length.

Acknowledgment. K.C.M., E.J.R., and A.S. thank the Institute for Mathematics and its Applications (Minneapolis, MN, with funds provided by the National Science Foundation) for support during the thematic year: Mathematics of Molecular and Cellular Biology. K.C.M. also thanks the Centre de Mathematiques et d'Informatique (Marseille, France) for the hospitality during this work. A.S. was supported in part by Swiss National Science Foundation Grant 3100A0-116275.

\section{References and Notes}

(1) de Gennes, P. G. Scaling Concepts in Polymer Physics; Cornell University Press: Ithaca, NY, 1979.

(2) Deutsch, J. M. Phys. Rev. E 1999, 59, 2539-2541.

(3) Grosberg, A. Y. Phys. Rev. Lett. 2000, 85, 3858-3861.

(4) Shimamura, M. K.; Deguchi, T. Phys. Rev. E 2002, 65, 051802.

(5) Dobay, A.; Dubochet, J.; Millett, K.; Sottas, P.-E.; Stasiak, A. Proc. Natl. Acad. Sci. U.S.A. 2003, 100, 5611-5615.

(6) Diao, Y.; Dobay, A.; Kusner, R. B.; Millett, K. C.; Stasiak, A. J. Phys. A 2003, 36, 11561-11574.

(7) Moore, N. T.; Lua, R. C.; Grosberg, A. Y. Proc. Natl. Acad. Sci. U.S.A. 2004, 101, 13431-13435.

(8) Millett, K.; Dobay, A.; Stasiak, A. Macromolecules 2005, 38, 601606.

(9) Steinhauser, M. O. J. Chem. Phys. 2005, 122, 094901-1-13.

(10) Plunkett, P.; Piatek, M.; Dobay, A.; Kern, J.; Millett, K.; Stasiak, A.; Rawdon, E. Macromolecules 2007, 40, 3860-3867.

(11) Rawdon, E.; Dobay, A.; Kern, J. C.; Millett, K. C.; Piatek, M.; Plunkett, P.; Stasiak, A. Macromolecules 2008, 41, 4444-4451.

(12) des Cloizeaux, J. J. Phys., Lett. 1981, 42, L433-436.

(13) Shimamura, M. K.; Deguchi, T. J. Phys. A 2002, 35, 241-246.
(14) Prellberg, T. J. Phys. A: Math. Gen. 2001, 34, L599-L602.

(15) Kuhn, W. Colloid Polym. Sci. 1934, 68, 2-15.

(16) Zifferer, G.; Preusser, W. Macromol. Theory Simul. 2001, 10, 397407.

(17) Guo, L.; Luijten, E. Macromolecules 2003, 36, 8201-8204.

(18) Haber, C.; Ruiz, S. A.; Wirtz, D. Proc. Natl. Acad. Sci. U.S.A. 2000, 97, 10792-10795.

(19) Maier, B.; Rädler, J. O. Macromolecules 2001, 34, 5723-5724.

(20) Aronovitz, J. A.; Nelson, D. R. J. Phys. (Paris) 1986, 47, 1445-1456.

(21) Cannon, J. W.; Aronovitz, J. A.; Goldbart, P. J. Phys. I 1991, 1, 629645.

(22) Rudnick, J.; Gaspari, G. J. Phys. A: Math. Gen. 1986, 19, L191-L193.

(23) Theodorou, D. N.; Suter, U. W. Macromolecules 1985, 18, 12061214.

(24) Bishop, M.; Michels, J. P. J. J. Chem. Phys. 1986, 85, 1074-1076.

(25) Bishop, M.; Saltiel, C. J. J. Chem. Phys. 1988, 88, 3976-3980.

(26) Diehl, H. W.; Eisenriegler, E. J. Phys. A: Math. Gen. 1989, 22, L87L91.

(27) Alim, K.; Frey, E. Phys. Rev. Lett. 2007, 27-1-4.

(28) Millett, K. C. Knotting of regular polygons in 3-space. In Random Knotting and Linking (Vancouver, BC, 1993); World Sci. Publishing: Singapore, 1994; pp 31-46.

(29) Calvo, J. A.; Millett, K. C. Minimal edge piecewise linear knots. In Ideal Knots; World Sci. Publ.: River Edge, NJ, 1998; Vol. 19, pp $107-128$.

(30) Rawdon, E. J.; Scharein, R. G. Upper bounds for equilateral stick numbers. In Physical Knots: Knotting, Linking, and Folding Geometric Objects in R3 (Las Vegas, NV, 2001); Amer. Math. Soc.: Providence, RI, 2002; Vol. 304, pp 55-75.

(31) Sumners, D. W.; Whittington, S. G. J. Phys. A 1988, 21, 1689-1694.

(32) Pippenger, N. Discrete Appl. Math. 1989, 25, 273-278.

(33) Diao, Y.; Pippenger, N.; Sumners, D. W. J. Knot Theory Ramifications 1994, 3, 419-429, Random knotting and linking (Vancouver, BC, 1993).

(34) Diao, Y. J. Knot Theory Ramifications 1995, 4, 189-196.

(35) Wolfram Research, I. Mathematica, Version 6.0.0, Champaign, IL, 2007.

(36) Klenin, K. V.; Vologodskii, A. V.; Anshelevich, V. V.; Dykhne, A. M.; Frank-Kamenetskii, M. D. J. Biomol. Struct. Dyn. 1988, 5, 11731185.

(37) Freyd, P.; Yetter, D.; Hoste, J.; Lickorish, W. B. R.; Millett, K.; Ocneanu, A. Bull. Am. Math. Soc. 1985, 12, 239-246.

(38) Ewing, B.; Millett, K. C. Computational algorithms and the complexity of link polynomials. In Progress in Knot Theory and Related Topics; Hermann: Paris, 1997; pp 51-68.

(39) Casella, G.; George, E. I. Am. Stat. 1992, 46, 167-174.

(40) Orlandini, E.; Tesi, M. C.; Janse van Rensburg, E. J.; Whittington, S. G. J. Phys. A 1998, 31, 5953-5967.

(41) Katritch, V.; Olson, W. K.; Vologodskii, A.; Dubochet, J.; Stasiak, A. Phys. Rev. E 2000, 61, 5545-5549.

(42) Hastings, M. B.; Daya, Z. A.; Ben-Naim, E.; Ecke, R. E. Phys. Rev. E 2002, 66, 025102 .

(43) Marcone, B.; Orlandini, E.; Stella, A. L.; Zonta, F. J. Phys. A: Math. Gen. 2005, 38, L15-L21.

(44) Diao, Y.; Nardo, J. C.; Sun, Y. J. Knot Theory Ramifications 2001, 10, 597-607.

MA801389C 